\title{
Fabrication additive : état de l'art et besoins métrologiques engendrés
}

\section{Additive manufacturing: state of the art and generated metrological needs}

\author{
Anne-Françoise OBATON ${ }^{1}$, Alain BERNARD ${ }^{2,3}$, Georges TAILLANDIER ${ }^{3}$ et Jean-Marc MOSCHETTA ${ }^{1}$ \\ ${ }^{1}$ Laboratoire commun de métrologie LNE-CNAM (LCM), LNE, 1 Rue Gaston Boissier, 75724 Paris Cedex 15, France, anne-francoise.obaton@ @ne.fr. \\ 2 Ecole Centrale de Nantes, 1 Rue de la Noë, BP 92101, 44321 Nantes Cedex 3, France. \\ 3 AFPR, 5 Avenue du Maréchal Leclerc, 92360 Meudon-La-Forêt, France.
}

\begin{abstract}
Résumé
Cet article fait un état de l'art sur la technologie de «fabrication additive », vulgarisée sous l'intitulé «impression 3D », et recense les besoins en métrologie et essais qu'elle engendre. Il définit la fabrication additive et présente les matériaux utilisés, l'intérêt et les freins de la technologie ainsi que les domaines d'application. Il énumère et décrit également en détail le principe des sept catégories de procédés qu'elle regroupe.
\end{abstract}

MOTS CLÉS : FABRICATION ADDITIVE, IMPRESSION 3D, ESSAIS, METROLOGIE.

\begin{abstract}
This article is a review about "additive manufacturing", popularized under "3D printing", and takes an inventory of the tests and metrological needs that the technology generates. It defines additive manufacturing and presents the materials used, the significance and the brakes of the technology, and application areas. It also lists and describes in detail the principle of the seven categories of processes that the technology counts.
\end{abstract}

KEYWORDS: ADDITIVE MANUFACTURING, 3D PRINTING, TESTS, METROLOGY.

\section{Introduction}

Dans le grand public, la terminologie «impression $3 \mathrm{D} »$ est usitée. Cependant, celle adoptée par les comités de normalisation est, "Additive Manufacturing" (AM) en anglais pour l'international (ISO/TC $261^{1}$ ) et « Fabrication Additive » (FA) pour la France (UNM 920²) [1].

La première technique de FA qui a vu le jour est la stéréolithographie. Elle a été inventée en France, par JeanClaude André, Alain Le Mehauté et Olivier De Witte, et brevetée sous le nom de «Dispositif pour réaliser un modèle de pièce industrielle », pour le compte de la société Cilas-Alcatel [2]. Le brevet a été déposé le 16 juillet 1984 tandis que l'américain Charles W. Hull déposait le sien, sur la même technique, le 8 août 1984 [3]. Charles W. Hull fondait, en 1986, la première société (3D Systems) pour commercialiser le procédé de stéréolithographie.

Il faudra attendre 1997 pour qu'une société française, Optoform (rachetée en 2001 par 3D Systems), co-fondée par André-Luc Allanic et Philippe Schaeffer, se lance dans la fabrication et la commercialisation de machines. Aujourd'hui, la France ne compte plus que deux sociétés fabricantes de machines à usage professionnel : Prodways (Phidias Technologies créée en 2008 devenue Prodways en 2013), du groupe Gorgé, et BeAM (créée en 2012), l'américain 3D Systems ayant racheté la troisième, Phenix Systems, en 2013.

\footnotetext{
${ }^{1}$ ISO/TC 261 : Commission de normalisation internationale sur la FA de l'International organization for standardization (ISO).

2 UNM 920 : Commission de normalisation sur la FA de l'Union de normalisation de la mécanique (UNM).
} 
La FA n'est donc pas une technique nouvelle, les industriels l'utilisent, depuis plus de 20 ans, principalement pour du prototypage $[4,5]$. L'ampleur actuelle de sa médiatisation vient du fait que des brevets de machines de FA viennent d'expirer, ouvrant ainsi la porte à la commercialisation de machines pour le grand public. Cet engouement a aussi pour conséquence de sensibiliser les industriels à l'utilisation de ce procédé pour la production d'objets fonctionnels en petites séries. Or, toute nouvelle application d'une technologie dans l'industrie fait apparaître des besoins en métrologie et essais.

Depuis plus de vingt ans (1992) l'Association Française de Prototypage Rapide (AFPR) rassemble les partenaires du prototypage rapide et aujourd'hui de la FA. Elle favorise la formation et le transfert de la technologie dans les entreprises. De part son statut, elle est parfaitement au fait des procédés et du marché de la FA.

Le Laboratoire national de métrologie et d'essais (LNE), quant à lui, est le laboratoire de référence chargé d'accompagner les industriels dans leur évolution et leurs progrès pour améliorer la compétitivité de leur entreprise en assurant la qualité et la conformité de leurs produits. En tant que tel, le LNE s'est efforcé de recenser les besoins en métrologie et essais que la technologie engendre, dans le but de développer les références (étalons ou méthodes de mesure) et les bancs de tests nécessaires aux industriels.

Dans cet article, nous listons la nomenclature, de manière aussi exhaustive que possible, associée à la FA : chacun des termes est défini et le principe des différents procédés est donné. Nous évoquons également l'intérêt et les freins de la technologie, les matériaux utilisés ainsi que les domaines d'application de la FA. Enfin, nous énumérons les besoins généraux en métrologie et essais dans le domaine de la FA.

\section{Fabrication additive (FA)}

\subsection{Définition}

La fabrication additive regroupe les procédés permettant de fabriquer des pièces, par ajout successif de couches de matière, à partir d'un modèle numérique, sans recourir à de l'outillage [1]. Le terme « additive» s'oppose aux principes des méthodes traditionnelles, comme l'usinage... qui consistent à enlever de la matière. Ainsi, par opposition, on peut regrouper ces méthodes plus communes sous le vocable «fabrication soustractive » [6].

\subsection{Matériaux}

Selon les procédés, les matériaux utilisés se présentent principalement sous forme de liquide, de poudre, de filament ou de feuille et peuvent être des plastiques, des métaux, de la céramique, du papier, du bois, des cires, des composites (ex. : des charges métalliques ou de céramique sont ajoutées au plastique pour améliorer sa rigidité) ou encore des biomatériaux. Un éventail des matériaux utilisés est donné dans le tableau 1.
Tableau 1

Liste des principaux matériaux utilisés en FA [7].

\begin{tabular}{|c|c|c|c|}
\hline Métaux & Plastiques & Céramiques & $\begin{array}{l}\text { Matériaux } \\
\text { organiques }\end{array}$ \\
\hline Aluminium & $\begin{array}{l}\text { Acrylonitrile } \\
\text { butadiène } \\
\text { styrène }(\mathrm{ABS})\end{array}$ & Alumine & Cires \\
\hline Acier & $\begin{array}{l}\text { Acide } \\
\text { polylactique } \\
\text { (PLA) }\end{array}$ & Mullite & $\begin{array}{l}\text { Tissus/ } \\
\text { cellules }\end{array}$ \\
\hline Titane & $\begin{array}{l}\text { Polyamide } \\
\text { (nylon) }\end{array}$ & Zircone & \\
\hline Inconel & $\begin{array}{l}\text { Polyamide } \\
\text { renforcé }\end{array}$ & $\begin{array}{l}\text { Cerbure } \\
\text { de silicium }\end{array}$ & \\
\hline $\begin{array}{l}\text { Cobalt- } \\
\text { chrome }\end{array}$ & $\begin{array}{l}\text { Polyétheréther } \\
\text { cétone } \\
(\text { PEEK) }\end{array}$ & $\begin{array}{l}\text { Phosphate } \\
\text { triçalcique } \beta\end{array}$ & \\
\hline Or/platine & $\begin{array}{l}\text { Polyméthacrylate } \\
\text { de méthyle } \\
\text { (PMMA) }\end{array}$ & Silice (sable) & \\
\hline Hastelloy & $\begin{array}{l}\text { Polycarbonate } \\
(\mathrm{PC})\end{array}$ & Plâtre & \\
\hline \multirow[t]{4}{*}{ Cuivre } & $\begin{array}{l}\text { Polyphénylsulfone } \\
\text { (PPSU ou PPSF) }\end{array}$ & Graphite & \\
\hline & $\begin{array}{l}\text { Résine époxy } \\
\text { thermodurcissables }\end{array}$ & $\begin{array}{l}\text { Résines } \\
\text { époxy } \\
\text { chargées en } \\
\text { céramique } \\
\text { (nano) }\end{array}$ & \\
\hline & Alumide & & \\
\hline & Ultern & & \\
\hline
\end{tabular}

* Inconel : alliage contenant une grande quantité de nickel et de chrome (environ $8250 \mathrm{~kg} \cdot \mathrm{m}^{-3}$ ).

\subsection{Intérêt et limites au développement}

La FA n'est pas «la» technologie qui va éclipser toutes les autres (usinage, moulage, découpage, injection, formage, fonderie, façonnage, frittage, soudure, forge). C'est une technique de fabrication complémentaire à celles déjà existantes, permettant notamment la réalisation de nouveaux types de pièces.

\subsubsection{Avantages du procédé de FA}

Les avantages de la technologie sont multiples [6-16] et les principaux sont listés ci-dessous :

- liberté de formes : la technologie permet de réaliser des pièces de forme extrêmement complexe (fig. 1), irréalisables par des techniques traditionnelles (notamment des canaux internes);

- allégement des pièces : la complexité des formes peut être utilisée pour alléger des pièces déjà existantes par optimisation topologique ${ }^{3}$ (fig. 2);

- réparation de pièces (fig. 3);

3 P. Bauer du groupe Thalès définit l'optimisation topologique comme « la bonne matière, la bonne quantité, au bon endroit et pour la bonne raison » [17]. 


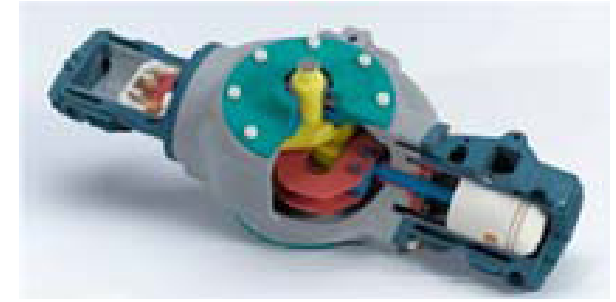

Fig. 1. - Exemples de pièces complexes réalisées en FA (société 3D Systems).

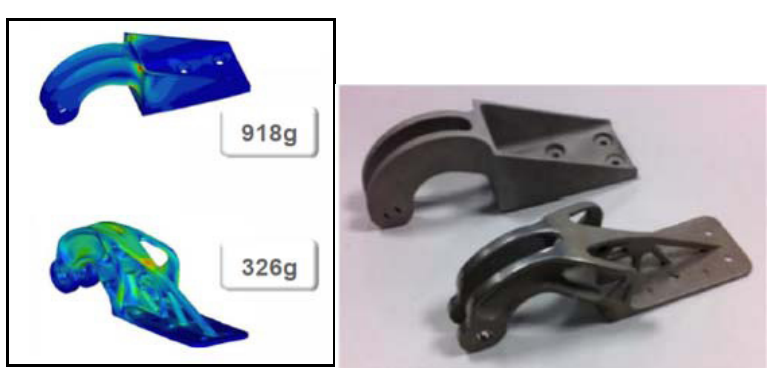

Fig. 2. - Exemple d'optimisation topologique d'une charnière réalisée en FA (société Multistation pour Airbus Group Innovations).

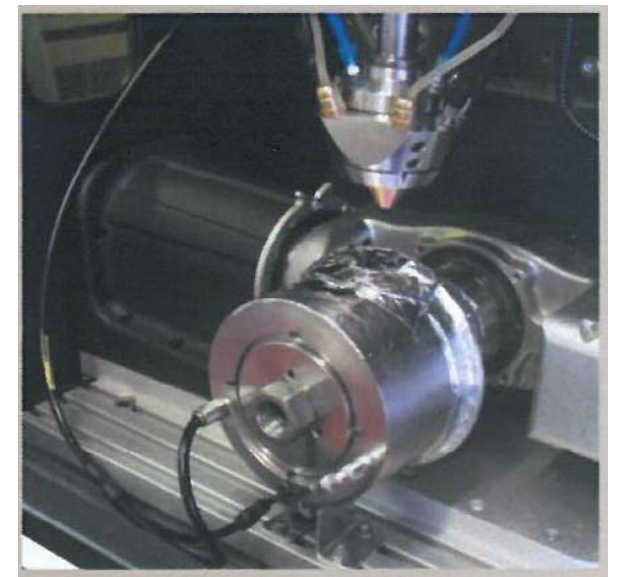

Fig. 3. - Procédé CLAD de la société BeAM utilisé, sur la photographie, pour la réparation d'une pièce métallique.

- personnalisation : la technique permet de réaliser du sur mesure (prothèses personnalisées dans le secteur médical ou dentaire, outillage de production comme des moules) [18];

- suppression des assemblages et des soudures : les pièces, même celles qui sont articulées (fig. 4), sont réalisées en une seule étape (gain de temps et limitation des risques d'endommagement aux jonctions des éléments);

- économie de matière : en fin de processus de fabrication, la matière non utilisée est recyclable pour une utilisation ultérieure ;

- pas d'outillage : la technologie ne nécessite pas l'utilisation d'outils spécifiques; elle est autonome;

- intégration de plusieurs fonctions dans un seul objet;
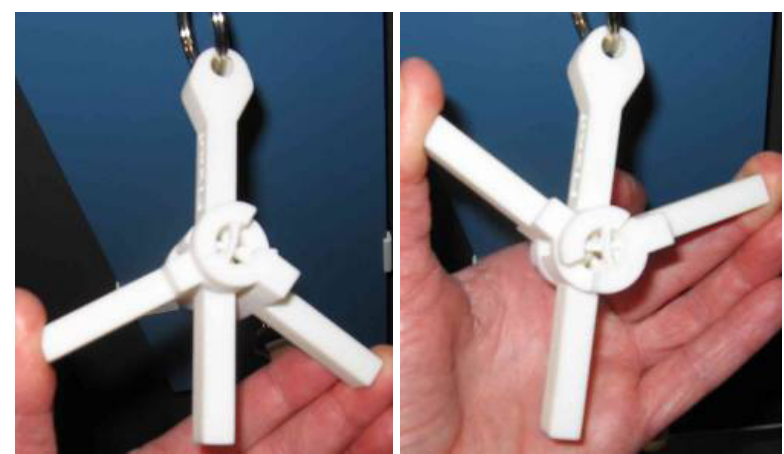

Fig. 4. - Exemple de pièces articulées réalisées en FA (société Materialise).

- proximité de l'utilisateur final ;

- écologique et économique : les pièces de rechange peuvent être imprimées localement par simple envoi du modèle numérique (pas de transport, gain de place et minimisation des pertes) $[8,19]$;

- production d'une grande variété de pièces avec une seule et même imprimante;

- pas de corrélation directe entre la complexité d'un objet et son coût ;

- production à la demande.

Cependant, à l'heure actuelle, le procédé ne présente pas que des avantages.

\subsubsection{Limitations technologiques au développement de la FA dans l'industrie}

Les acteurs de le FA s'accordent pour dire que le marché, dans ce domaine, est en très forte croissance mais pas encore mature. La principale limitation au développement de ce procédé provient de la matière première. Les industriels se plaignent de ne pas disposer de suffisamment de matériaux pour satisfaire la demande et reprochent aux fabricants de machines de contraindre l'utilisateur à n'utiliser que les matériaux qu'ils commercialisent. Pourtant, certains fabricants, à l'image d'EOS et de Prodways, ouvrent leur système.

Les autres principales limitations de la FA émanent du temps d'impression et du coût des machines. Cependant, les facteurs suivants représentent également des freins pour les industriels :

- compétences plurielles : cette technologie nécessite des compétences multiples d'où la lenteur des industriels à l'intégrer dans leur processus de fabrication. Il est nécessaire d'étendre les compétences mobilisées à des acteurs connaissant les processus de numérisation et de modélisation à partir d'informations discrètes issues de cette numérisation;

- état de surface de l'objet final : l'état de surface des pièces en fin de fabrication est mauvais (rugueux, effet escalier) et le post-traitement nécessite plus de temps que dans le cas de l'usinage; 


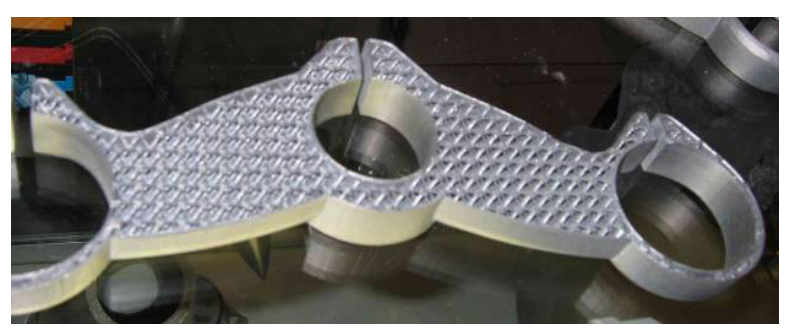

Fig. 5. - Exemple de structure alvéolaire (société Poly-Shape).

- manque de fiabilité : les procédés ne sont pas encore suffisamment répétables;

- petites séries uniquement : la FA n'est pas adaptée pour satisfaire de gros volumes de production.

\subsection{Domaines d'application}

La FA concerne de nombreux domaines d'application dans divers secteurs d'activités : aéronautique, aérospatial, automobile, dentaire et médical mais également horlogerie, bijouterie (fig. 6), sport [20], design et mode.

Dans le monde industriel, la FA est essentiellement utilisée pour du prototypage mais les industries, concernées par la production de petites séries, commencent à l'utiliser pour fabriquer des pièces fonctionnelles [21]. Notamment, la FA est utilisée pour réaliser des structures alvéolaires (fig. 5) encore appelées structures « lattices» pour alléger des pièces mais également pour dissiper la chaleur. Par ailleurs, la réparation de pièces existantes est également un domaine dans lequel la FA excelle (fig. 3). En ce qui concerne le prototypage, elle apporte un gain substantiel de temps et d'argent sur l'ensemble du cycle de vie du produit (de la conception au produit final) en permettant de matérialiser rapidement le produit en cours de développement avant le lancement de sa production en grande série [22].

L'utilisation de la FA dans le domaine médical et dentaire est très développée en France. Dans le secteur médical, la FA permet de réaliser des implants orthopédiques à façon [23] (prothèse de hanche...) et d'améliorer l'ergonomie de prothèses (prothèses auditives [24]...). Dans le secteur dentaire, elle permet la fabrication en masse de prothèses personnalisées.

Nous citons ici quelques exemples d'utilisation de la FA dans l'industrie.

La société Cirtes réalise, par stratification de couches, des aménagements d'avions pour le compte d'Airbus [25].

La société Poly-Shape dispose de quinze machines de FA pour travailler sur des projets d'envergure. Elle réalise notamment, pour l'aérospatiale, des pièces pivots, d'une grande légèreté, pour des panneaux solaires implantés sur des satellites [17].

De grands groupes industriels, comme PSA, utilisent essentiellement la FA, non pas en production, mais dans leurs bureaux d'étude et dans leurs ateliers pour du prototypage. PSA privilégie l'aspect visuel et la tenue dans le temps de l'objet fini. La technique (extrusion de matière avec du polycarbonate, polyphenylsulfane et de l'ABS) leur permet de réaliser des maquettes à une échelle réduite traitées en finition pour le perçu, des maquettes technologiques pour expliquer une innovation (ex. : culasse, armature de siège) et de l'équipement de véhicules «concept car» (ex. : casquette, écran). PSA utilise également la FA pour optimiser son planning (montage à blanc d'une copie de pièces fonctionnelles pour valider le montage). Il fabrique cependant, en petites séries, quelques pièces fonctionnelles sur lesquelles ne s'exerce aucune contrainte de sécurité [17].

Le groupe Safran, concerné par les petites séries (une quinzaine de moteurs d'avion à livrer par an) utilise la FA (fusion sur lit de poudre (laser et faisceau d'électrons), dépôt de matière sous énergie concentrée) pour réaliser des pièces fonctionnelles de moteur d'avion et de fusée. La technologie lui permet d'alléger les avions en optimisant la topologie des pièces fabriquées [17].

Par ailleurs, beaucoup de ces groupes disposent de Fablab. Ces structures mettent à la disposition de tout le monde (aux employés de l'entreprise mais également à des personnes extérieures) des machines de fabrication additive. D'après M. Desmoulins, «open innovation manager » chez Renault, cela leur permet d'une part de repérer les personnes prédisposées à cette technologie et d'autre part de partager des idées [17].

\subsection{Catégories et principes des procédés}

Il existe une multitude de méthodes de fabrication additive. Elles sont toutes basées sur la fabrication de couches successives de matière à partir d'un modèle numérique mais diffèrent au niveau des matériaux de base, ainsi que la forme sous laquelle ces matériaux se présentent, la technique de fabrication d'une couche et comment ces couches sont liées les unes aux autres. Par ailleurs, certains de ces procédés nécessitent des supports de construction (fig. 6) pour pouvoir combler les zones de vides et permettre la dépose d'une couche sur la précédente. Ces supports sont imprimés en parallèle et sont enlevés en fin de fabrication au cours du post-traitement. Ils engendrent des restrictions géométriques sur le dessin de la pièce, afin de pouvoir les retirer. Le post-traitement peut également comprendre, dans certains procédés, une exposition de l'objet fini à un rayonnement pour parfaire la réaction chimique ayant permis la solidification d'une couche et accroître la résistance de l'objet. Enfin, une amélioration de l'état de surface de l'objet en fin de fabrication peut s'avérer nécessaire.

Au cours des années, les techniques ont évolué et de nouvelles sont apparues si bien que leur classement a évolué également [26]. Elles ont été classées selon la caractéristique des états initial/final ${ }^{4}$ du matériau de base $[4,26]$

\footnotetext{
${ }^{4}$ États initial/final : liquide/solide, solide/solide ou poudre/ solide.
} 


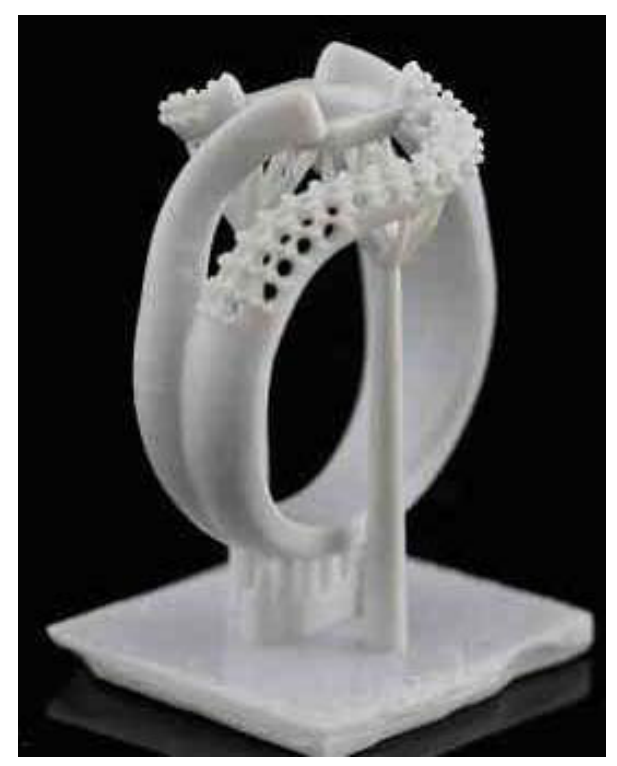

Fig. 6. - Bague et supports réalisés en ABS par un procédé de la catégorie "photopolymérisation en cuve par lampe avec un photomasque » (société Delta Technologie).

puis par technologie $[9,10]$. La terminologie et la définition des différentes catégories de procédés est en cours de normalisation aux niveaux national (UNM 920²) et international (développement conjoint ISO/ASTM ${ }^{5}$ ). Nous reprenons donc le classement des différentes catégories de procédés, au nombre de sept (tableau en Annexe), tel qu'il est défini dans la norme NF ISO 17296-2 [27]. Le principe de base de chacune de ces catégories de procédé est décrit dans ce paragraphe et récapitulé dans l'Annexe de l'article.

Les toutes premières étapes de tous ces procédés sont identiques. Elles comprennent :

1) conception d'un modèle numérique décrivant la géométrie de l'objet à fabriquer, avec un logiciel approprié [28];

2) conversion du modèle numérique en modèle $\mathrm{STL}^{6}$ (format de fichier standard pour la FA). Le format STL permet de discrétiser la surface de l'objet sous forme d'un maillage polyédrique à facettes triangulaires (fig. 7);

3) prévision des supports éventuels;

4) découpage du modèle STL en strates (de quelques dizaines de micromètres à plusieurs millimètres d'épaisseur) représentant les couches à imprimer;

5) transfert du fichier à la machine de FA ;

6) paramétrages de la machine de FA.

\footnotetext{
5 ASTM : American Society for Testing and Materials.

${ }^{6}$ STL : STL, pour STéréoLithographie, est le format de fichier originel du logiciel de $\mathrm{CAO}$ associé au procédé de stéréolithographie créé par la société 3D Systems. STL est également connu sous le nom «Standard tessellation language ».
}

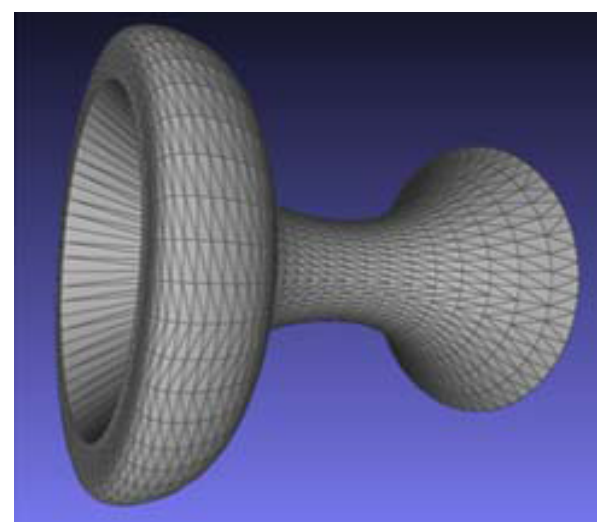

Fig. 7. - Exemple d'objet en modèle STL.

\subsubsection{Photopolymérisation en cuve (vat photopolymerization)}

\subsubsection{Par laser point par point}

Ce type de machine comprend une cuve remplie de photopolymères liquides. Dans cette cuve est immergée une plateforme de translation, suivant l'axe vertical, sur laquelle sera réalisée la pièce à fabriquer. La machine est également équipée d'un mécanisme de recouvrement et de nivellement de la surface du liquide, d'un laser, généralement UV, et d'un miroir permettant de focaliser le faisceau laser sur la surface du liquide. La fabrication se déroule conformément aux étapes suivantes :

1) immersion de la plateforme dans la cuve de photopolymères ;

2) balayage point par point, suivant le modèle numérique, avec le laser, de la surface du liquide $\Rightarrow$ photopolymérisation de la première couche (durcissement) et, si nécessaire, de la structure du support;

3) descente de la plateforme d'une épaisseur de couche ;

4) recouvrement et nivellement de la surface du liquide ;

5) balayage point par point, suivant le modèle numérique, avec le laser, de la surface du liquide $\Rightarrow$ photopolymérisation de la deuxième couche et, si nécessaire, de la structure du support;

6) ces étapes se répètent jusqu'à ce que le modèle soit créé ;

7) nettoyage, suppression des supports, postpolymérisation par une nouvelle exposition UV.

La figure 8 décrit schématiquement les étapes du procédé de stéréolithographie appartenant à cette catégorie de procédés.

\subsubsection{Par lampe avec un photomasque}

Ce type de machine comprend une cuve remplie de photopolymères liquides. Le fond de cette cuve est 


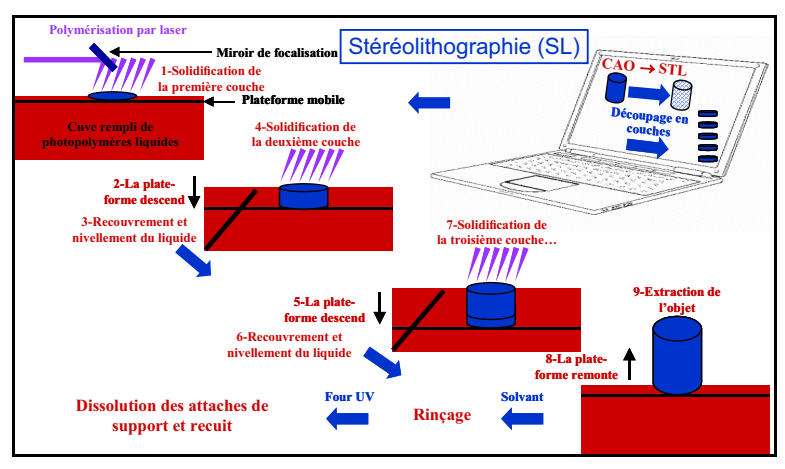

Fig. 8. - Schématisation des étapes du procédé de stéréolithographie (SL ou SLA) appartenant à la catégorie «photopolymérisation en cuve par laser point par point ».

généralement transparent. Une plateforme de translation, suivant l'axe vertical, est immergée dans la cuve. Elle permet un accrochage, par le dessus, de la pièce à fabriquer. L'intérêt de cette configuration est de réduire le volume de photopolymères liquides nécessaire, donc la cuve. En contrepartie, ces machines sont réservées uniquement à la réalisation de petites pièces. La machine est également équipée d'un écran à diodes électroluminescentes $\left(\mathrm{LED}^{7}\right)$ qui permet de réaliser un photomasque (seules les LED reproduisant le modèle numérique d'une couche sont éclairées). La fabrication se déroule conformément aux étapes suivantes :

1) immersion de la plateforme dans la cuve de photopolymères ;

2) projection lumineuse de l'image de la couche sur le liquide avec le photomasque, suivant le modèle numérique $\Rightarrow$ photopolymérisation de la première couche et, si nécessaire, de la structure du support;

3) remontée de la plateforme d'une épaisseur de couche ;

4) projection lumineuse de l'image de la couche sur le liquide avec le photomasque, suivant le modèle numérique $\Rightarrow$ photopolymérisation de la deuxième couche et, si nécessaire, de la structure du support;

5) ces étapes se répètent jusqu'à ce que le modèle soit créé ;

6) nettoyage, suppression des supports, postpolymérisation par une nouvelle exposition UV.

La technologie MOVINGLight, industrialisée par la société française Prodways, se range dans cette catégorie. Cependant, le principe du photomasque est différent et l'accrochage de la pièce se fait par le dessous. La technologie combine une source de lumière à base de $\mathrm{LED}^{7} \mathrm{UV}$ $(365 \mathrm{~nm})$ à forte puissance et une tête d'impression comprenant des puces micro-électroniques (DLP : Digital Light Processing). Ces puces se composent d'une matrice contenant jusqu'à 2 millions de miroirs microscopiques orientables (fig. 9). La tête se déplace d'une zone

\footnotetext{
${ }^{7}$ LED : Light-Emitting Diode.
}

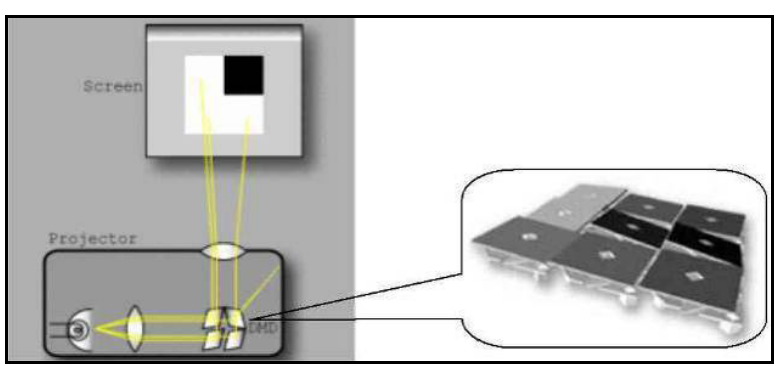

Fig. 9. - Principe d'une imprimante de FA de la catégorie «photopolymérisation en cuve par lampe avec un photomasque » [9]. Seulement 4 miroirs orientables sont représentés (DMD).

d'impression à l'autre. Chaque miroir représente un pixel de l'image à projeter. Suivant son orientation, il illumine ou pas la surface de photopolymères. La photopolymérisation d'une couche est réalisée en une seule étape. Lorsque la matière liquide exposée est durcie, le processus se répète. Prodways propose des polymères fortement chargés en céramique ou métal, et, dans un futur proche, en fibre ou nano-particules pour améliorer les propriétés mécaniques des objets fabriqués par leur procédé. Les machines qu'il conçoit sont principalement destinées aux domaines dentaire et médical.

\subsection{2. $\quad$ Projection de matière (material jetting)}

Ce type de machine comprend une plateforme de translation, suivant l'axe vertical, sur laquelle reposera la pièce à fabriquer. Elle comprend également, au-dessus de la plateforme un appareil distributeur de photopolymère liquide ou de cire fondue ainsi qu'une source de rayonnement lumineux fixée à la tête d'impression. La fabrication se déroule conformément aux étapes suivantes :

1) dépôt sélectif, suivant le modèle numérique, de fines gouttelettes de matériau photosensible $\Rightarrow$ fabrication de la première couche et, éventuellement, de la structure du support;

2) photopolymérisation du liquide ou solidification de la résine avec une lampe UV;

3) descente de la plateforme d'une épaisseur de couche ;

4) dépôt sélectif, suivant le modèle numérique, de fines gouttelettes de matériau photosensible $\Rightarrow$ fabrication de la deuxième couche et, éventuellement, de la structure du support;

5) ces étapes se répètent jusqu'à ce que le modèle soit créé ;

6) nettoyage, suppression des supports, postpolymérisation par une nouvelle exposition UV.

La figure 10 décrit schématiquement les étapes du procédé de multi-jet modelling (MJM) appartenant à cette catégorie de procédés. 


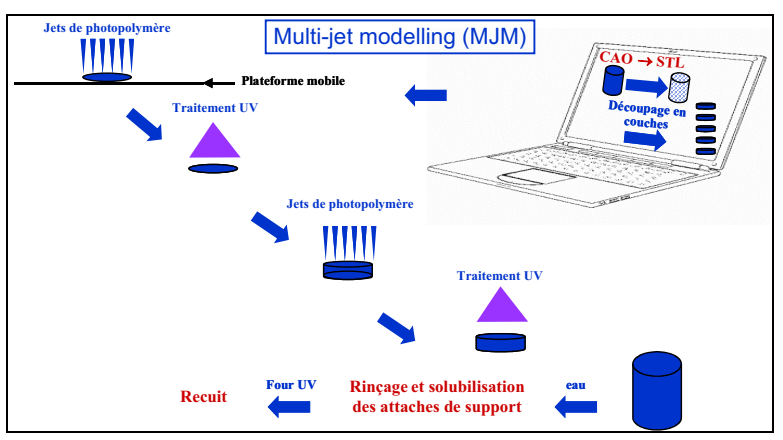

Fig. 10. - Schématisation des étapes du procédé «multi-jet modelling $(M J M)$ » appartenant à la catégorie «projection de matière».

\subsubsection{Projection de liant (binder jetting)}

Ce type de machine comprend une cuve équipée d'une plateforme de translation, suivant l'axe vertical, remplie de poudre. Un dispositif d'étalement de la poudre permet d'homogénéiser la surface de poudre entre chaque couche. Un appareil distributeur d'agent de liant liquide est inclus dans la tête d'impression. La fabrication se déroule conformément aux étapes suivantes :

1) répartition de la poudre;

2) dépôt sélectif de fines gouttes de liant, suivant le modèle numérique, pour solidifier la poudre $\Rightarrow$ création de la première couche;

3) descente du bac de poudre d'une épaisseur de couche ;

4) uniformisation de la surface de poudre;

5) dépôt sélectif de fines gouttes de liant, suivant le modèle numérique, pour solidifier la poudre $\Rightarrow$ création de la deuxième couche;

6) ces étapes se répètent jusqu'à ce que le modèle soit créé ;

7) nettoyage et suppression de la poudre excédentaire.

\subsubsection{Fusion sur lit de poudre (powder bed fusion)}

\subsubsection{Par laser point par point}

Ce type de machine comprend un système d'alimentation en poudre et un dispositif d'étalement permettant d'homogénéiser la surface de poudre entre chaque couche. La machine est également équipée d'un laser et d'un miroir permettant de focaliser le laser sur la surface de la poudre. La fabrication se déroule conformément aux étapes suivantes :

1) chauffage de la poudre en dessous de la température de fusion. Le laser ne fait qu'ajouter un petit différentiel d'énergie pour le frittage ${ }^{8}$ ou la fusion;

\footnotetext{
${ }^{8}$ Frittage : sous l'effet de la chaleur du laser les grains se soudent entre eux sans pour autant atteindre le point de fusion.
}

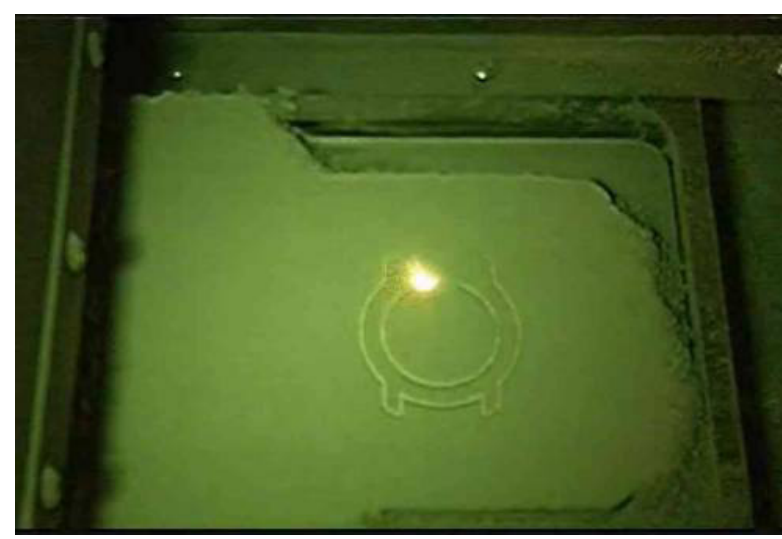

Fig. 11. - Photographie du procédé de «frittage sélectif par laser (FSL) » appartenant à la catégorie « fusion sur lit de poudre par laser point par point » (société Phenix Systems du groupe 3D Systems).

2) uniformisation de la poudre;

3) balayage sélectif du lit de poudre, suivant le modèle numérique, avec le laser $\Rightarrow$ frittage $\Rightarrow$ création de la première couche et des supports uniquement pour la fabrication avec des poudres métalliques;

4) descente du bac de poudre d'une épaisseur de couche;

5) dépôt d'une couche de poudre pour recouvrir la couche précédente;

6) balayage sélectif du lit de poudre, suivant le modèle numérique, avec le laser $\Rightarrow$ frittage $\Rightarrow$ création de la deuxième couche et des supports uniquement pour la fabrication avec des poudres métalliques ;

7) ces étapes se répètent jusqu'à ce que le modèle soit créé ;

8) suppression de la poudre excédentaire ;

9) suppression des supports pour la fabrication avec des poudres métalliques;

10) amélioration de l'état de surface.

La figure 11 présente une photographie de la fabrication d'une couche dans une machine appartenant à la catégorie «fusion sur lit de poudre par laser point par point ».

La société Phenix Systems, entreprise française rachetée par l'entreprise américaine 3D Systems en 2013, fabrique et commercialise des machines basées sur ce principe. Ces machines utilisent des poudres céramiques (alumine) et métalliques (aciers inox, aciers outillages, alliages non-ferreux, super alliages, métaux précieux). Elles sont principalement utilisées pour la production dans le domaine dentaire. 


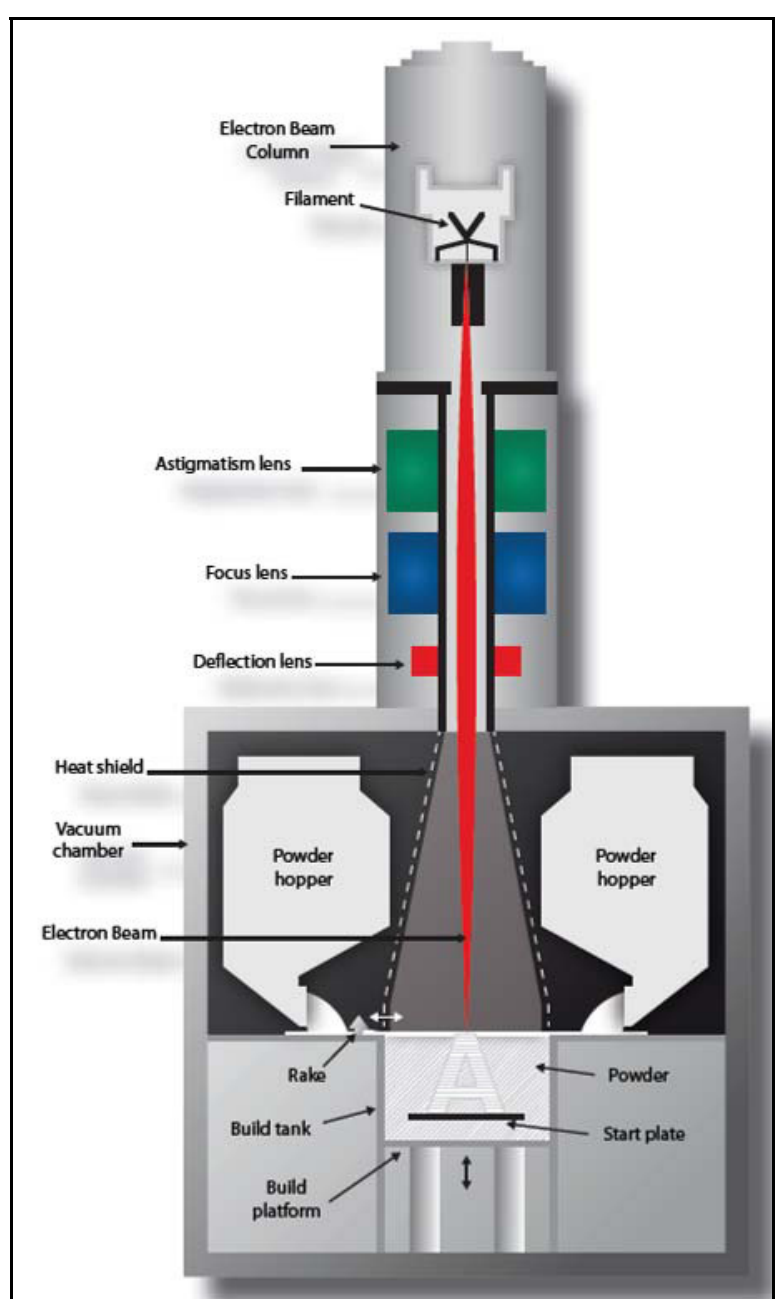

Fig. 12. - Schéma d'une imprimante de FA utilisant un procédé de la catégorie «fusion sur lit de poudre par faisceau d'électrons » (société Arcam).

\subsubsection{Par faisceau d'électrons point par point}

Le principe de ce procédé de fabrication (fig. 12) est analogue à celui par laser. La seule différence réside dans la source d'activation remplacée par un faisceau d'électrons focalisé.

\subsubsection{Par lampe infrarouge à travers un masque}

Ce type de machine comprend une cuve équipée d'une plateforme de translation, suivant l'axe vertical, remplie de poudre. Un dispositif d'étalement de la poudre permet d'homogénéiser la surface de poudre entre chaque couche. La machine est également équipée d'une source de radiations infrarouge, d'une plaque de verre et d'un distributeur d'encre en poudre. La fabrication se déroule conformément aux étapes suivantes :

1) impression à l'encre (méthode similaire à celle utilisée dans un photocopieur), suivant le modèle numérique, de la première couche, en négatif, sur la plaque de verre $\Rightarrow$ création du masque représentant la première couche;

2) uniformisation de la poudre ;

3) mise en place du masque au-dessus de la cuve de poudre;

4) exposition à la source de radiations infrarouge, à travers le masque, de la cuve de poudre $\Rightarrow$ frittage $\Rightarrow$ création de la première couche, en une seule opération ;

5) nettoyage de la plaque de verre pour supprimer le masque correspondant à la première couche ;

6) impression à l'encre, suivant le modèle numérique, de la seconde couche, en négatif, sur la plaque de verre $\Rightarrow$ création du masque représentant la deuxième couche;

7) descente du bac de poudre d'une épaisseur de couche ;

8) dépôt d'une couche de poudre pour recouvrir la couche précédente;

9) mise en place du masque au-dessus de la cuve de poudre;

10) exposition à la source de radiations infrarouge, à travers le masque, de la cuve de poudre $\Rightarrow$ frittage $\Rightarrow$ création de la deuxième couche, en une seule opération;

11) ces étapes se répètent jusqu'à ce que le modèle soit créé ;

12) suppression de la poudre excédentaire;

13) amélioration de l'état de surface.

La figure 13 décrit schématiquement les étapes du procédé de « selective mask sintering (SMS) » appartenant à cette catégorie de procédés.

\subsubsection{Extrusion de matière (material extrusion)}

Ce type de machine (fig. 14) comprend généralement une plateforme de translation, suivant l'axe vertical (z), et une buse chauffée, positionnée sur une tête d'impression mobile dans le plan $(x y)$, permettant la distribution de matière pour fabriquer l'objet. Certaines machines sont équipées d'une deuxième buse pour la fabrication du support. La fabrication se déroule conformément aux étapes suivantes :

1) ramollissement de la matière dans la tête d'impression ;

2) distribution sélective de filaments de matière ramollie à travers la buse, suivant le modèle numérique $\Rightarrow$ création de la première couche et, éventuellement, des supports ; 


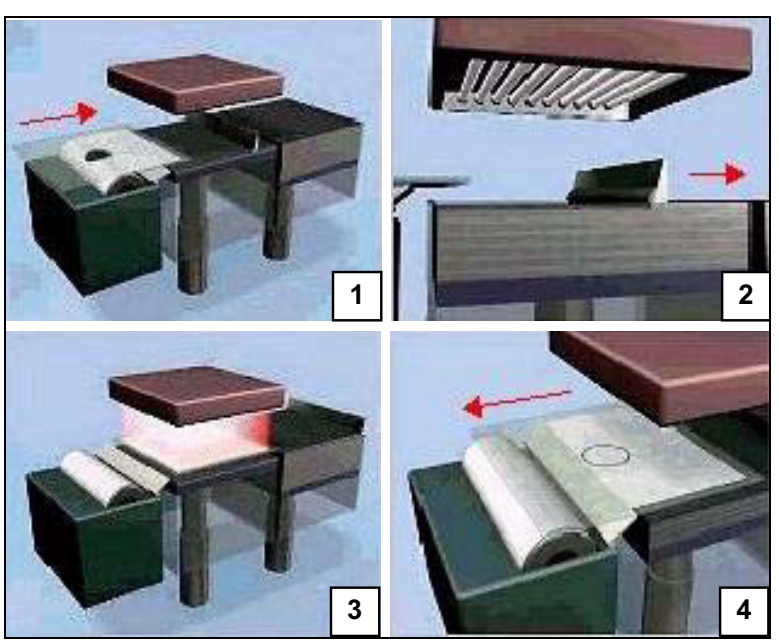

Fig. 13. - Illustrations schématiques de quatre étapes d'impression avec une imprimante de FA utilisant un procédé de la catégorie «fusion sur lit de poudre par lampe infrarouge à travers un masque » [29].

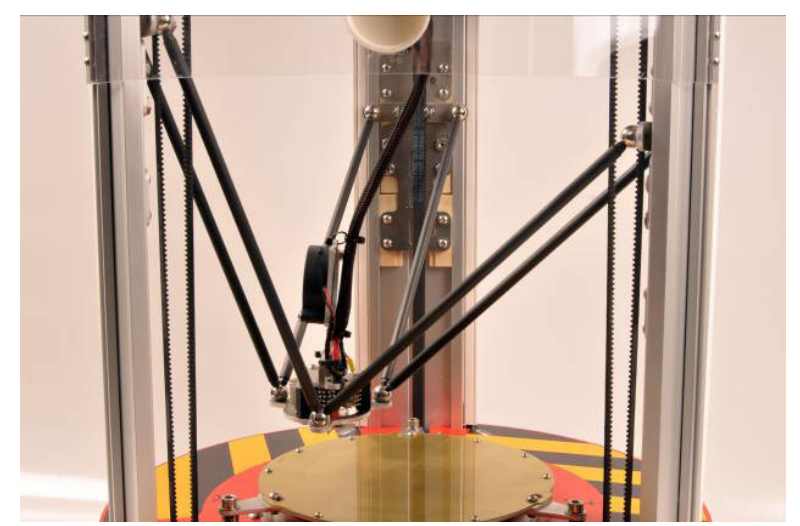

Fig. 14. - Photographie d'une imprimante de FA utilisant un des procédés de la catégorie «extrusion de matière » (société Spiderbot, fabricant français). Dans ce cas de figure la tête d'impression est montée sur un système hexapode et la plateforme de fabrication est fixe.

3) solidification de la couche par refroidissement;

4) descente de la plateforme d'une épaisseur de couche;

5) distribution sélective de filaments de matière ramollie à travers la buse, suivant le modèle numérique $\Rightarrow$ création de la deuxième couche et, éventuellement, des supports;

6) ces étapes se répètent jusqu'à ce que le modèle soit créé;

7) suppression des supports éventuels;

8) amélioration de l'état de surface.
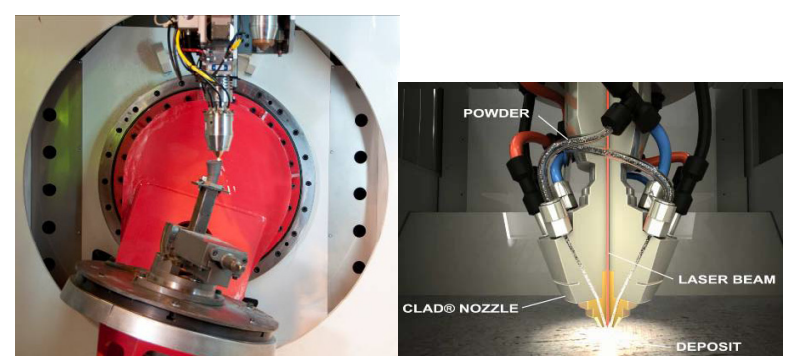

Fig. 15. - Photographie et schéma de la tête d'impression d'une imprimante de FA utilisant un des procédés de la catégorie "dépôt de matière sous énergie concentrée » (société BeAM).

\subsubsection{Dépôt de matière sous énergie concentrée (directed energy deposition)}

\subsubsection{Par laser point par point}

Ce type de machine (fig. 15) comprend une plateforme de translation et une tête d'impression orientable. La tête d'impression se déplace linéairement suivant trois axes orthogonaux et la plateforme sur laquelle est fixé l'objet à fabriquer, ou à réparer, suivant deux axes de rotation (lacet et roulis). La tête d'impression inclue une source d'énergie thermique focalisée sur une buse dans laquelle transite de la poudre ou un filament métallique. La matière est fondue dans la buse au fur et à mesure, avant d'être déposée sélectivement. La fabrication se déroule conformément aux étapes suivantes :

1) fusion et dépôt sélectif de la matière, suivant le modèle numérique $\Rightarrow$ création de la première couche;

2) fusion et dépôt sélectif de la matière, suivant le modèle numérique $\Rightarrow$ création de la deuxième couche;

3) ces étapes se répètent jusqu'à ce que le modèle soit créé;

4) amélioration de l'état de surface.

La société française BeAM fabrique des machines classées dans cette catégorie. Le procédé de base de leur technologie, dénommée «CLAD» pour «Construction Laser Additive Directe », a été développé, à l'origine, par le centre de transfert technologique Irepa Laser. La matière déposée est protégée localement ou globalement de l'oxydation par un gaz neutre. Leur technique permet de fabriquer des pièces fonctionnelles en métal (alliages d'inconel, aciers inox, alliages de titane, multi matériaux) (fig. 15) mais également de réparer des pièces métalliques (fig. 3). Cette technologie est particulièrement utilisée dans l'aéronautique.

\subsubsection{Par arc plasma transféré}

Le principe de ce type de machine est analogue à celui par laser. La seule différence réside dans la source d'activation remplacée par un arc plasma transféré. 


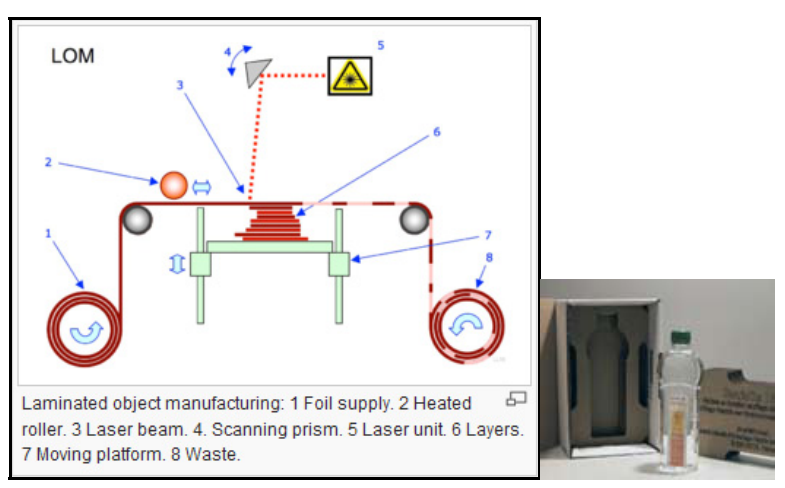

Fig. 16. - Imprimante de FA utilisant un des procédés de la catégorie «stratification de couches » (société Cirtes).

\subsubsection{Stratification de couches (sheet lamination)}

\subsubsection{Par rouleau continu}

Ce type de machine (fig. 16) comprend une table de translation, suivant l'axe vertical, un dispositif de déroulement de matière (papier, polymère) composé de deux rouleaux positionnés de part et d'autre de la table, un dispositif de découpe (laser, cutter...) et un rouleau chauffant de laminage. La fabrication se déroule conformément aux étapes suivantes :

1) découpe du matériau suivant le modèle numérique $\Rightarrow$ création de la première couche;

2) dépose d'un adhésif;

3) alimentation en matière ;

4) collage par réaction chimique et thermique par passage du rouleau chauffant de laminage ;

5) découpe du matériau suivant le modèle numérique $\Rightarrow$ création de la deuxième couche ;

6) ces étapes se répètent jusqu'à ce que le modèle soit créé ;

7) suppression des déchets.

\subsubsection{Par rouleau discontinu}

Ce type de machine comprend trois plateformes de translation au lieu d'une comme dans le procédé précédent, suivant l'axe vertical : une pour la fabrication et les deux autres, réparties de part et d'autre de la première, pour l'alimentation en feuilles de matière (métal). La machine est également équipée d'un dispositif de découpe (laser) et d'une source ultrasonore. La fabrication se déroule conformément aux étapes suivantes :

1) alimentation en matière ;

2) découpe du matériau suivant le modèle numérique $\Rightarrow$ création de la première couche;
3) alimentation en matière ;

4) soudure par ultrason;

5) découpe du matériau suivant le modèle numérique $\Rightarrow$ création de la deuxième couche;

6) ces étapes se répètent jusqu'à ce que le modèle soit créé;

7) suppression par fraisage des déchets.

\subsubsection{Par mise en panoplie}

Le principe de ce procédé repose sur l'usinage ou la découpe de chaque couche à partir d'une plaque par mise en panoplie suivi de l'assemblage de ces couches avec un positionnement grâce à des inserts (principe de la stratoconception ${ }^{\circledR}$ ). Le maintien en position des couches est réalisé par différents procédés : collage, brasage, bridage mécanique, etc.

\section{Besoins en métrologie et contrôles associés à la FA}

De nombreux échanges avec des acteurs de la FA : fabricants de machines, prestataires de service, revendeurs de machines, institutions académiques travaillant sur la technologie et industriels utilisant la FA pour réaliser des pièces, nous ont permis d'identifier que la technologie plastique est essentiellement utilisée pour fabriquer des prototypes ou pour réaliser des pièces fonctionnelles sur lesquelles il n'y a pas d'exigence de sécurité. En revanche, des besoins en contrôle qualité de pièces métalliques et en céramique, et en qualification de machines de fabrication émergent notamment pour les secteurs médical, aéronautique et aérospatial. Cependant, la caractérisation de pièces réalisées en plastique peut également s'avérer nécessaire. Les besoins recensés en matière de mesures et essais associés à la FA sont listés cidessous. Ceux-ci se répartissent dans un large éventail de compétences.

\subsection{Côté matériaux}

\subsubsection{Analyse des poudres}

Afin que les pièces réalisées, avec les procédés utilisant des poudres, soient de bonne qualité, il est nécessaire de pouvoir établir la traçabilité des matériaux à l'aide de certificats d'étalonnage et d'effectuer des analyses en termes de :

- dimensionnement des grains (la taille des grains doit être comprise entre $20 \mu \mathrm{m}$ et $30 \mu \mathrm{m}$ );

- forme des grains (sphéricité, diamètre);

- distribution de la taille des grains;

- composition chimique ; 
- qualité des poudres non-utilisées avant et après la fabrication.

\subsubsection{Certification de nouveaux matériaux}

Tous les professionnels utilisant les procédés de FA s'accordent à dire qu'il n'y a pas suffisamment de matériaux disponibles sur le marché pour couvrir tous leurs besoins et souhaitent que de nouveaux matériaux soient certifiés.

\subsection{3. Étude de la loi de comportement des matériaux}

Afin d'évaluer les performances mécaniques des objets fabriqués, des essais de traction et de fatigue doivent être réalisés sur des éprouvettes. Ces essais permettent notamment d'adapter le traitement thermique des pièces finies afin d'améliorer leurs propriétés mécaniques. Ils sont couramment demandés pour évaluer la résistance mécanique des structures alvéolaires.

\subsection{4. $\quad$ Analyse de la ductilité des matériaux}

Ce type d'analyse peut être pratiqué avec un microscope électronique à balayage (MEB) sur des éprouvettes. Cette ductilité dépend notamment de l'orientation de fabrication de la pièce.

\subsection{Côté pièces fabriquées}

\subsubsection{Mesure de la géométrie externe}

Ce type de mesures est couramment réalisée dans le domaine avec des scanners 3D. Cependant, des machines à mesurer tridimensionnelles peuvent également être utilisées pour des mesures plus exigeantes en termes d'incertitudes sur la mesure, si l'état de surface de la pièce, en termes de rugosité, et la géométrie le permettent.

\subsection{2. $\quad$ Mesure de la géométrie interne}

Des besoins se font ressentir sur le contrôle de l'épaisseur des objets et sur leur dimensionnement en $3 \mathrm{D}$ volumique.

\subsubsection{Contrôle qualité}

Recherche de défauts internes, contrôle des structures «lattices » et de poudre de fabrication non-fusionnée. En effet, les utilisateurs des procédés à base de poudre, souhaitent s'assurer qu'il ne reste pas de poudre non-utilisée et de poudre non-fondue dans leurs structures alvéolaires.

\subsubsection{Contrôle de l'exactitude des procédés de FA}

Dans la catégorie «projection de liant», les dimensions des objets fabriqués dans le plan $x y$ sont très souvent sous-dimensionnées alors que la dimension dans la direction $z$ est surdimensionnée. Par ailleurs, les trous sont toujours sous-dimensionnés, et une bosse est présente au fond de tous les trous [12].

\subsubsection{Caractérisation de l'état de surface des pièces finales}

En fin de fabrication l'état de surface des pièces présente des aspérités. Des mesures en terme de rugosité et de porosité peuvent s'avérer nécessaires afin d'évaluer les post-traitements à réaliser. L'état de surface des pièces est dépendant de leur orientation par rapport au sens de fabrication dans la machine.

\subsubsection{Essais au feu des pièces fabriquées}

Certaines pièces réalisées en plastique et ignifugées nécessitent de subir des essais au feu.

\subsubsection{Pérennité et traçabilité dans le temps}

Le vieillissement des matériaux est mal connu. Or, cela peut s'avérer problématique pour les orthèses et les prothèses dans le médical et les pièces critiques dans l'aéronautique et l'aérospatial.

\subsection{Côté machines}

\subsubsection{Qualification de machines avec un nouveau matériau}

Le fabricant de machine qualifie la machine avec ses matériaux. En cas de changement de matériau, la machine devra être qualifiée à nouveau.

\subsubsection{Contrôle in situ de la fabrication de chaque couche}

Des contraintes importantes s'exercent sur les couches lors de la fabrication. Elles se trouvent alternativement dans différents états extrêmes : fusion/ refroidissement/fusion,... En conséquence, les utilisateurs de la FA souhaiteraient que la fabrication de chaque couche soit contrôlée en ligne, dans la machine, afin d'ajuster automatiquement son paramétrage pour compenser des déformations (retrait matière) sur une couche au niveau de la couche suivante dans le but d'arriver au niveau de qualité requis. Polyshape réalise ce type de contrôle, actuellement, par prise de photographies à chaque couche, associée à une analyse d'image. D'autres méthodes peuvent être envisagées. 


\subsubsection{Surveillance de la puissance laser pendant la fabrication}

Lors de la fabrication simultanée de plusieurs pièces identiques, d'une zone d'impression à une autre, les pièces réalisées ne sont pas similaires. Ce phénomène est attribué aux hétérogénéités de température dans les enceintes des machines de FA impliquant des lasers. Il faudrait donc cartographier la température dans l'enceinte et asservir la puissance du laser pour réguler la température.

\subsubsection{Qualification des lasers pour les procédés concernés} laser.

Elle porte sur l'étalonnage de la taille du faisceau

\subsubsection{Caractérisation et qualification des machines pour améliorer les procédés}

Il faut que le processus de fabrication soit répétable lors de la réalisation simultanée d'une série de pièces identiques, et reproductible lors de la réalisation consécutive d'une même pièce. Par conséquent, dans un premier temps, il faut étudier la répétabilité et la reproductibilité du processus de fabrication sur des pièces finies. Dans un second temps, il faut identifier les paramètres qui influent sur la fabrication (facteurs d'influence : température, taille du faisceau laser, localisation de la pièce dans l'enceinte de fabrication...), estimer leur poids et, si possible, minimiser les facteurs prépondérants.

\subsection{Côté appareils de mesure}

\subsection{1. Étalonnage et traçabilité de scanner 3D}

Les scanners 3D sont beaucoup utilisés en parallèle de la FA pour 3 raisons principales :

- le balayage d'une pièce dans le but de la reproduire (rétro-conception);

- l'emballage sur mesure d'une pièce en utilisant le procédé de stratification de couches ;

- le balayage de la pièce finale afin d'effectuer des comparaisons avec le modèle numérique initial.

\subsubsection{Caractérisation et qualification de tomographe à rayon $X$}

Ce type de dispositif est actuellement essentiellement utilisé pour la recherche de défauts et relativement peu pour la mesure dimensionnelle. En conséquence, pour cette nouvelle application, il est nécessaire de caractériser et de qualifier ces tomographes en concevant des objets de référence (étalons), en définissant les méthodes de raccordement de ces étalons et en définissant les séries de mesures à faire avec ces étalons ainsi que les différents paramètres machine à faire varier.

\subsubsection{Recherche de méthodes alternatives à la tomographie à rayon $\mathrm{X}$}

À l'heure actuelle, la recherche de défauts internes et de poudre de fabrication non-fusionnée, les mesures densimétriques et de la géométrie interne des objets fabriqués ou d'échantillons ou d'éprouvettes fabriqués simultanément sont réalisées par tomographie ${ }^{9}$ à rayons X. Ces mesures sont généralement externalisées par les prestataires de services. Ce type de prestations augmente considérablement le coût de la pièce. En conséquence, les acteurs de la FA souhaiteraient que des méthodes alternatives soient proposées.

\subsection{Côté normalisation}

Comme pour toutes technologies émergentes, il faut définir la terminologie et fixer des règles d'usage en considérant tous les aspects de la FA.

\section{Conclusion}

Cet article réalise un état de l'art sur la fabrication additive (FA) afin de recenser les besoins en mesures et essais qu'engendre cette technique très médiatisée actuellement.

Dans la première partie, est abordée la FA : sa définition, les avantages et les limitations, les sept différents procédés que regroupent cette technologie, le type et la forme des matériaux utilisés et les domaines d'application de la FA. Il est souligné notamment que cette technologie perce de façon significative dans les secteurs industriel et médical pour la production d'objets fonctionnels en petite quantité et pour la production en masse de pièces personnalisées après avoir été essentiellement destinée aux prototypes.

Dans une seconde partie, sont énumérés les besoins en mesures et essais associés à la FA.

Compte-tenu de ces besoins identifiés, le département dimensionnel du LNE a entrepris, dans un premier temps, d'explorer de nouvelles méthodes de mesure dimensionnelle en volume pour caractériser des pièces réalisées en FA. Par ailleurs, le LNE s'implique dans la normalisation sur la FA et prévoit, dans un second temps, de s'impliquer dans la caractérisation et la qualification de tomographes à rayon $\mathrm{X}$ et de machines de FA.

\section{Remerciements}

Les auteurs remercient Florence Coullon, Arnaud Fenichel et Fabienne Carpentier, du centre de documentation du LNE, pour leur aide inestimable apportée à la recherche bibliographique.

9 La tomographie est une technique de contrôle non destructif permettant d'imager en 3D la structure interne d'un objet en le sondant avec des ondes électromagnétiques ou sonores sous différents angles de vue. La tomographie à rayons $\mathrm{X}$ est la technique la plus répandue. 


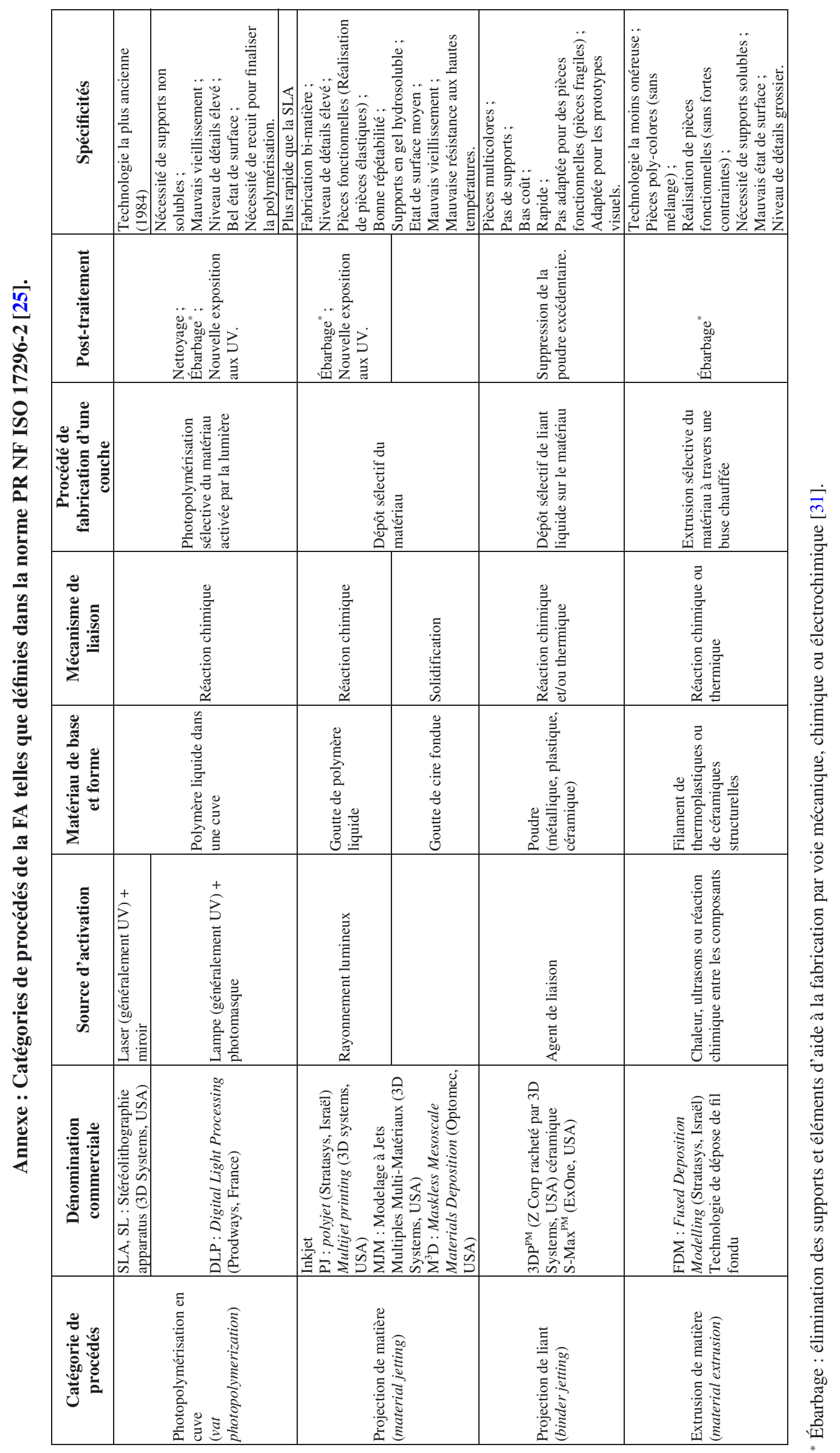




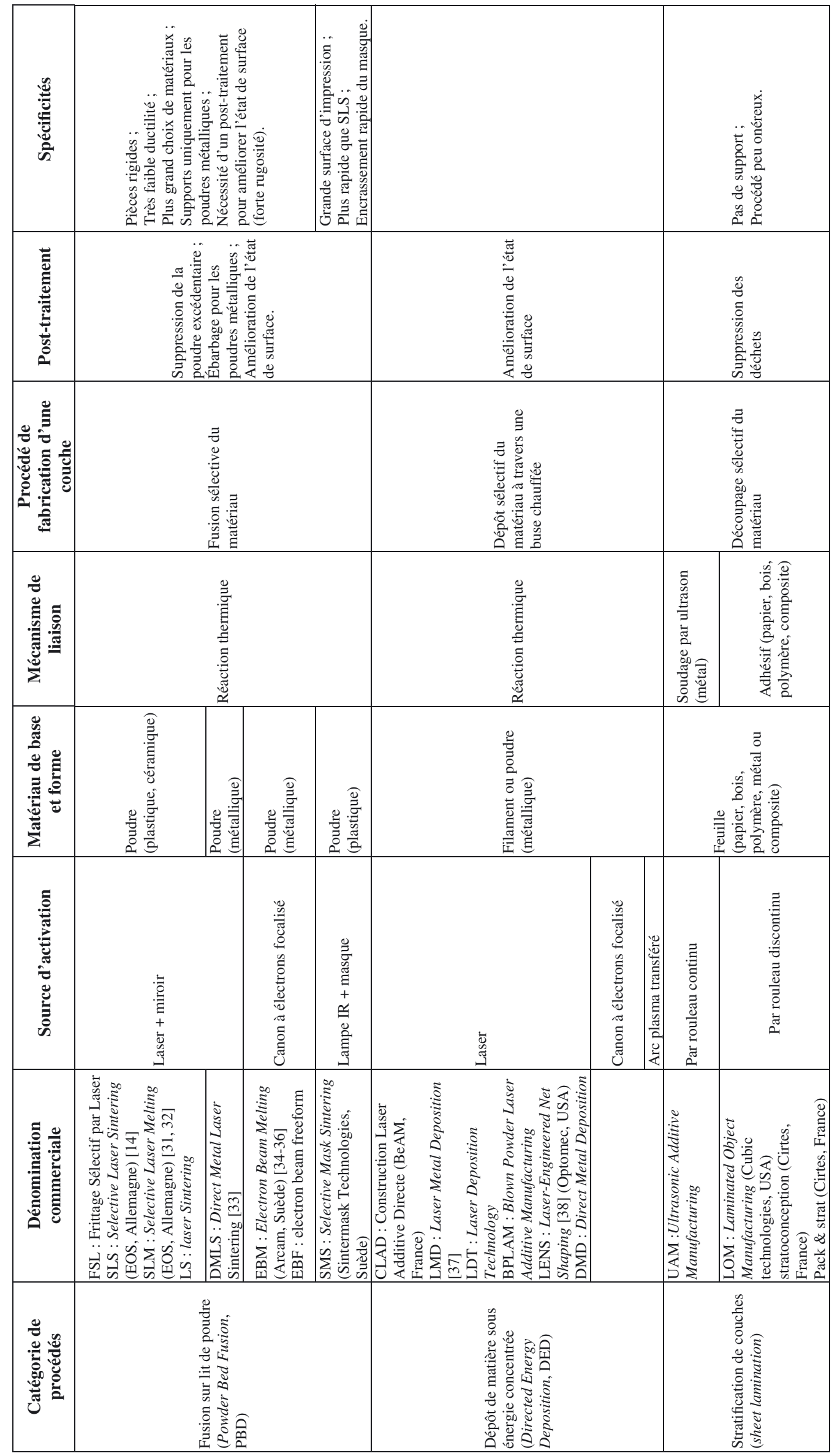




\section{Références}

[1] AFNOR, NF E 67-001, 2011, « Fabrication additiveVocabulaire ».

[2] André J.-C., Le Mehauté A. et De Witte O., « Dispositif pour réaliser un modèle de pièce industrielle », Brevet FR 2567668 - A1, Date de dépôt : 1607-1984, date de publication : 17-01-1986.

[3] Hull C.W., "Method and apparatus for production of three-dimensional objects by stereolythography", Brevet EP 0171069 - A2, Date de dépôt : 08-08-1984, date de publication : 12-02-1986.

[4] Dubois P., Aoussat A. et Duchamp R., «Prototypage rapide - Généralités », Dossier Techniques de l'Ingénieur, l'expertise technique et scientifique de référence, BM7017, 10/04/2000.

[5] BeRnaRd A. et TAILlandiER G., « Le prototypage rapide », Ed. Hermès, 1998.

[6] GUO N. et LEU M.C., "Additive manufacturing: technology, applications and research needs", Front. Mech. Eng., 8, 3, 2013, 215-243.

[7] BERCHON M. et BerTIER L., «L'impression 3D », Serial makers, Eyrolles, 2013, EAN13 : 782212135220.

[8] BRANS K., "3D printing, a maturing technology", 11th IFAC Workshop on Intelligent Manufacturing, 2013.

[9] NAssiRY A., «Fabrication additive de pièces en polymères », Rapport Plastipolis, 28 octobre 2013.

[10] Wohlers report: Additive manufacturing and 3D printing state of the industry; annual worldwide progress report / Tbv1Wohlers Associates; ID: gnd/6014226-1. Fort Collins, Colorado: Wohlers Associates, 2012, ISBN: 0975442988.

[11] LIN S.L., LIN C.C., LIN D.Y. et ChUANG C.S., "Laser additive manufacturing technology in titanium 64 implant of microstructure fabrication and analysis", 8th Annual IEEE International Conference on Nano/Micro Engineered and Molecular Systems, IEEE NEMS 2013, 2013, 594-597.

[12] Islam M.N., Boswell B., et PramaniK A., "An investigation of dimensional accuracy of parts produced by three-dimensional printing", Lect. Notes Eng. Comput. Sci., 1 LNECS, 2013, 522-525.

[13] Meredith B., Morham B. et Gonzalez P., "Application of 3D printing for small UAVs", Int. SAMPE Technical Conference, 2013, 16-33.

[14] Deckers J., Kruth J.-P., CARdon L., Shahzad K. et VLEUGELS J., "Densification and geometrical assessments of alumina parts produced through indirect selective laser sintering of alumina-polystyrene composite powder", J. Mech. Eng., 59, 11, 2013, 646-661.

[15] RASMUSSEN R., "3D printed electronics: The future is now”, SMT Surface Mount Technology Magazine, 28, 62013, 8-11.

[16] MonTFORT-Windels F., rapport du Sirris, « Additive manufacturing nouveauté 2012-2013», juillet 2013.

[17] Salon 3D Print «Les rencontres de la fabrication additive », Lyon, France, 17-20 juin 2014.
[18] BANKS J., "Adding value in additive manufacturing: Researchers in the United Kingdom and Europe look to 3D printing for customization", IEEE Pulse, 4, 6, 2013, 22-26.

[19] CHEN Y., LI K. et QIAN X., "Direct geometry processing for tele-fabrication", Proceedings of the ASME Design Engineering Technical Conference, 2 (PARTS A AND B), 2012, 411-424.

[20] Manoharan V., Chou S.M., Forrester S., Chai et G.B.b, KoNG P.W., "Application of additive manufacturing techniques in sports footwear", Virtual and Physical Prototyping, 8, 4, 2013, 249-252.

[21] Bernard A., TAILlandier G. et Karunakaran K.P., "Evolution of Rapid Product Development with Rapid Manufacturing: Concepts and Applications", International Journal of Rapid Manufacturing, 1/1, 2009, 3-18, ISSN (Online) : 1757-8825, ISSN (Print) : 1757-8817 (Inderscience).

[22] Meredith B., Morham B. et Gonzalez P., "Application of 3D printing for small UAVs", Int. SAMPE Technical Conference, 2013, 16-33.

[23] Cronskär M., BÄCKströM M. et RÄNNAR L.-E., "Production of customized hip stem prostheses - A comparison between conventional machining and electron beam melting (EBM)", Rapid Prototyping J., 19, 5, 2013, 365-372.

[24] ManNOOR M.S., JiAng Z., JAMES T., Kong Y.L., Malatesta K.A., Soboyejo W.O., Verma N., Gracias D.H. et MCALPINE M.C.a, "3D printed bionic ears", Nano Lett., 13, 6, 2013, 2634-2639.

[25] $19^{\text {es }}$ Assises européennes de prototypage rapide, Association Française de Prototypage Rapide (AFPR), Châtenay-Malabry, France, 24-26 juin 2014.

[26] Gibson I., Rosen D.W. et Stucker B., "Additive Manufacturing Technologies: Rapid Prototyping to Direct Digital Manufacturing”, Springer, New York Heidelberg Dordrecht London, 2010.

[27] PR NF ISO 17296-2, «Fabrication additive-Principes généraux-Partie 2 : Vue d'ensemble des catégories de procédés et des matières premières », mai 2014. ISO/DIS 17296-2, "Additive manufacturing-General principles-Part 2: Overview of process categories and feedstock", 2014-06-24.

[28] ZHANG Y. et BERNARD A., "Grouping parts for multiple parts production in Additive Manufacturing", Science Direct, Procedia CIRP 17, 2014, 308-313.

[29] http://www.additive3d.com/pow/pow29.htm.

[30] XP E 67-30 Norme AFNOR, «Fabrication additive-Pièces réalisées par fabrication additive-Cahier des charges et conditions de réception », 15 mai 2013.

[31] Król M., DobrzańsKi L.A., Reimann Ł. et CzAJA I., "Surface quality in selective laser melting of metal powders", Archives Mater. Sci. Eng., 60, 2, 2013, 87-92.

[32] Strano G., Hao L., Everson R.M. et Evans K.E., "Surface roughness analysis, modelling and prediction in selective laser melting", J. Mater. Process. Technol., 213, 4, 2012, 589-597. 
[33] Calignano F., Manfredi D., Ambrosio E.P., IUliano L., et FINO P., "Influence of process parameters on surface roughness of aluminum parts produced by DMLS", Int. J. Adv. Manufact. Technol., 67, 9-12, 2013, 2743-2751.

[34] RaWAl S., Brantley J. et KarabudaK N., "Additive manufacturing of Ti-6Al-4V alloy components for spacecraft applications", RAST 2013 - Proceedings of 6th International Conference on Recent Advances in Space Technologies, 2013, 5-11.

Article reçu le 24 novembre 2014, version révisée reçue le 26 janvier 2015.
[35] DinWIDDIE R.B., DEHOFF R.R., LlOYd P.D., Low L.E. et ULRICH J.B., "Thermographic in-situ process monitoring of the electron beam melting technology used in additive manufacturing", Proceedings of SPIE - The International Society for Optical Engineering, 8705, 2013.

[36] Edwards P., O'ConNer A. et Ramulu, M, "Electron beam additive manufacturing of titanium components: Properties and performance", J. Manufact. Sci. Eng. Trans. ASME, 135, 6, 2013.

[37] Rombouts M., Maes G., Mertens M. et Hendrix W., "Laser metal deposition of Inconel 625: Microstructure and mechanical properties", J. Laser Appl., 24, 5, 2012.

[38] Balla V.K., Das M., Bose S., Janaki Ra G.D. et MANNA I.d, "Laser surface modification of 316 L stainless steel with bioactive hydroxyapatite", Mater. Sci. Eng. C, 33, 8, 2013, 4594-4598. 